\title{
La calidad en el sector servicios: El reto para la competitividad turística del Ecuador, experiencias de su aplicación
}

\section{Quality in the services sector: The challenge for Ecuador's tourism competitiveness, experiences of its application}

\author{
Walter Omar Ocaña Zambrano \\ Jenny Maribel Osejo Angulo \\ Universidad Tecnológica Equinoccial, Ecuador
}

Autor para correspondencia: walter.ocana@ute.edu.ec; jenny.osejo@ute.edu.ec Fecha de recepción: 15 de diciembre 2017 - Fecha de aceptación: 26 de febrero de 2018

Resumen: La calidad en la dinámica comercial actual se convierte en el principal factor diferenciador en un mercado cada vez más competitivo, más aún en el sector servicios, en donde el creciente surgimiento de nuevos destinos emergentes y la diversificación constante de productos turísticos, ha desarrollado una delgada línea entre la oferta y la decisión de compra o retorno por parte del cliente. En el Ecuador, este es un elemento que ha cobrado cada vez mayor relevancia en los últimos años, la implementación de normas y modelos de calidad se ha concentrado principalmente en los establecimientos orientados hacia un mercado receptivo, así como en los principales destinos de país, sin embargo, al proponerse una política pública orientada hacia el fomento de la calidad turística, el lenguaje de la calidad se ha ido incluyendo progresivamente en otros territorios y mercados. Este trabajo recoge algunas experiencias obtenidas en la aplicación de la calidad en el sector servicios, como parte de un proceso de formación y articulación con el sector empresarial, principalmente en pequeñas y medianas empresas turísticas y de la hospitalidad.

Palabras claves: calidad en el servicio; SERVQUAL; gestión de la calidad en turismo y hospitalidad

\begin{abstract}
The quality of the current commercial dynamics becomes the main differentiating factor in an increasingly competitive market, even more in the services sector, where the growing emergence of new emerging destinations and the constant diversification of Tourist products, has developed a thin line between the offer and the decision of purchase or return by the client. In Ecuador, this is an element that has become more and more relevant in recent years, the implementation of quality standards and models has been concentrated mainly in the establishments oriented towards a receptive market, as well as in the main Destinations in the country, however, as a public policy aimed at promoting tourism quality is being put in place, the language of quality has been progressively included in other territories and markets. This work includes some experiences obtained in the application of quality in the services sector, as part of a process of formation and articulation with the business sector, mainly in small and medium-sized tourism companies and hospitality.
\end{abstract}

Key words: quality in service; SERVQUAL; quality management in tourism and hospitality 


\section{Introducción}

El lenguaje de la calidad para el sector turístico y de la hospitalidad en el Ecuador, es un proceso reciente, si bien las primeras acciones encaminadas a su implementación iniciaron a parir de 2009, es en el año 2015, cuando cobra mayor fuerza al declararse a este como "el año de la calidad turística", otorgando el sello "Q" a 273 establecimientos a nivel nacional (Ministerio de Turismo, 2015). Cabe mencionar que, en ese mismo año se registraron en el país 25757 establecimientos en los cinco subsectores turísticos (intermediación, alojamiento, alimentación, recreación y transporte), siendo estos en su gran mayoría Mi Pymes (Mintur, 2015), es decir, apenas el $1.05 \%$ de los establecimientos a nivel nacional cuentan con algún tipo de reconocimiento respecto a la prestación de la calidad en sus servicios, situación compleja al considerar al turismo como parte de la política nacional de cara al cambio de la matriz productiva.

Del mismo modo, al ser el turismo y la hospitalidad uno de los sectores de mayor crecimiento a nivel mundial, uno de sus mayores retos se ubica en la mejora de la calidad como pieza clave para la consolidación de los destinos turísticos y su competitividad, es precisamente esta mejora principalmente en la cadena de valor de la prestación de los servicios, en donde la valoración de la satisfacción del cliente, define a la calidad misma, así pues, la calidad será entendida como el "resultado de una comparación subjetiva entre las expectativas previas de consumo y la percepción posterior a este” (Oliver, 1981, citado en Santomá \& Costa, 2007).

Es en este contexto que, el presente trabajo se orienta a identificar cómo se encuentra la implementación de la gestión de la calidad en el área de servicio de los establecimientos turísticos y de la hospitalidad, de manera particular se busca establecer cómo la relación entre una adecuada planeación estratégica organizacional, la implementación de procesos de operación y valoración del nivel de satisfacción del cliente, afectan de forma directa a la imagen de los establecimientos respecto a la percepción de la calidad, vista desde la perspectiva de los clientes, para ello el documento abordará los principios propuestos por Parasuraman, Zeithaml y Berry (1985), relacionados con el modelo Servqual para la valoración de la calidad en el servicio.

\section{Marco conceptual}

Para el estudio de la calidad, Garvin (1988) ha definido su evolución en cuatro grandes etapas: la primera denominada de "inspección", en donde el producto final era comparado con un estándar determinado, esta etapa corresponde al surgimiento de la revolución industrial, en donde la reducción de las pérdidas marco el desarrollo productivo de la naciente globalización de la economía; la segunda etapa denominada "control estadístico de la calidad", en donde efectivamente el control de la producción y la investigación estadística del ciclo de producción cobran fuerza y se institucionaliza en las principales empresas a nivel mundial; la tercera etapa corresponde al "aseguramiento de la calidad", para ello fue necesario comprobar que la inspección y medición de la calidad no son suficientes, sino que la planificación debe alcanzar a toda la organización; finalmente, la cuarta etapa corresponde a la "calidad como estrategia competitiva", la calidad se ubica en el más alto nivel directivo y se relaciona con la rentabilidad, se incluye y cobra fuerza el punto de vista del cliente y se lo ubica como eje central en la planificación estratégica empresarial, es este momento en donde la calidad es vista no solo como 
una estrategia para resolver dificultades y reducir los costos de producción de bienes [y servicios], sino que se convertirse en el principal valor diferenciador de las organizaciones en un mercado global altamente competitivo (Escobar y Mosquera, 2013).

Sin duda, un punto de quiebre en la evolución de la calidad ha sido la normalización y estandarización, pues estas siguen siendo el corazón de los procesos de la calidad, en la actualidad las normas internacionales ISO, esquematizan el modelo de calidad, la cual es definida como; "la promoción de una cultura que da como resultado comportamientos, actitudes, actividades y procesos para proporcionar valor mediante el cumplimiento de las necesidades y expectativas de los clientes [...]" (Secretaria Central de ISO, 2015). La satisfacción del cliente, el cumplimiento del valor percibido, el impacto previsto en todas las partes interesadas y fundamentalmente un enfoque basado en procesos, son los elementos en los que se consolida la gestión de la calidad propuesta desde de la Organización Internacional de Normalización (ISO, por sus siglas en inglés). A su vez, la articulación entre el sistema de gestión de la calidad (ISO 9001:2015), sistema de gestión ambiental (ISO 14011:2015) y el sistema de gestión en seguridad y salud en el trabajo (OHSAS 18001:2007), permiten dar un paso más allá en los llamados Sistemas de Gestión Integrados (SGI), que junto con los principios de sostenibilidad y responsabilidad social empresarial, se convierten en el último eslabón de los procesos de gestión empresarial en la actualidad.

Sin embargo, es en la prestación de servicios en donde las organizaciones compiten en el siglo veintiuno, pues difícilmente podemos encontrar un sector en donde los servicios no se encuentren presentes, tal es así, que en el sector industrial el servicio es visto como parte del producto (Miguel, 2002), pero yendo más allá, es necesario hablar de la gestión de la calidad en los servicios, pues hoy en día este sector ha cobrado tal relevancia que ya no puede ser solo visto como parte del proceso de producción, sino más bien ubicarse como parte propia de la gestión empresarial.

A su vez, como lo manifiesta Albacete (2004), existen claras diferencias en torno a la gestión de la calidad, respecto a la fabricación de productos y la prestación de servicio, pues si bien ambas buscan satisfacer las necesidades del cliente, la intangibilidad del servicio complica la valoración de sus resultados. Por otro lado, el límite entre producto y servicio podría ser superado cuando el producto es entendido como: "todo aquello que se ofrece en un mercado para atención, adquisición, uso o consumo y que puede satisfacer un deseo o necesidad. Incluye objetos físicos, servicios, personas, lugares, organizaciones e ideas" (Kotler, Bowen, Makens, Rufín, \& Reina, 2004).

A partir de lo cual, el servicio puede ser considerado no sólo como las actividades o procesos que se realizan en un negocio de forma intangible, sino, al conjunto o vínculo que se genera entre éste y el producto físico o bien tangible (Salazar Duque y Burbano Argoti, 2017). En otras palabras, el servicio es considerado como el "conjunto de prestaciones que el cliente espera recibir, además del producto” (López, 2003).

Es precisamente en esta visión más amplia del producto, en donde el sector del turismo y de la hospitalidad parecen haber eliminado el límite entre productos y servicios, pues durante su montaje-producción posee ambas características, siendo por ello necesario contar con un modelo 
de gestión específico (Santomá \& Costa, 2007). La calidad en el sector servicio es entendida como; la satisfacción del cliente, cuando este se siente satisfecho con el consumo de un producto o servicio como resultado de una comparación subjetiva entre las expectativas previas de consumo y la percepción posterior a este (Oliver, 1981, citado en Santomá \& Costa, 2007).

En tal virtud, para el análisis de la calidad en el servicio es necesario identificar las dos grandes escuelas de pensamiento, la norte europea y la norteamericana, la primera ubica tres principios básicos; la calidad técnica, que representa el servicio que los clientes han recibido, ello incluye los procesos previos a la prestación del servicio que incluye protocolos o normas internas de la organización; el segundo elemento hace referencia a la calidad funcional, es decir la forma en la que el servicio fue prestado por parte del personal en contacto con el cliente, es decir el cumplimiento con lo planificado; y finalmente, la imagen de la organización, que incluye la imagen de marca, las instalaciones y todos aquellos aspectos tangibles del servicio, sea esta analizada desde las expectativas del cliente o como la organización se presente ante el (Grönroos, 1984, citado en Santomá \& Costa, 2007).

Por otro lado, la escuela norteamericana es reconocida por el modelo Servqual, propuesto por Parasuraman, Zeithaml, \& Berry, quienes definen a la calidad en el servicio como; "la reducción o eliminación del desajuste entre las expectativas previas al consumo del servicio y la percepción del servicio prestado, medida a partir de la diferencia entre ambos conceptos" (1985). Para ello los autores ponen principal énfasis en la valoración desde la perspectiva del cliente a partir de cinco niveles que son; fiabilidad, capacidad de respuesta, seguridad, empatía, intangibilidad (Duque-Oliva y Parra Díaz, 2015).

Adicional a ello, son consideradas tres dimensiones complementarias para la valoración de la calidad en el servicio; siendo estas, la calidad física, que corresponde a los aspectos físicos del servicio, también denominados elementos tangibles; otra dimensión corresponde a la calidad corporativa, en este caso la imagen proyectada de la empresa, y finalmente, la calidad interactiva; centrada en la relación entre trabajadores y clientes y, clientes con clientes (Parasuraman, et al. 1985).

Finalmente, la experiencia de la calidad en el servicio será influenciada por otras dos dimensiones; la calidad técnica, relacionada con la correcta aplicación de los procesos (técnicas), para obtener una aceptación por parte del cliente; mientras que la calidad funcional, se enfoca en la forma de atención al cliente mientras se le brinda el servicio (Grönroos, 1994).

\section{Métodos}

El diseño de la investigación es de tipo descriptivo-exploratoria, con un enfoque cualitativo y empleando métodos como; observación no participante, sondeo de opinión y mapa de procesos o blueprint. Para el levantamiento de información se realizó una búsqueda de información en fuentes secundarias, a partir de textos académicos de bases indexadas, trabajos de titulación y documentos relacionados con los establecimientos analizados. Del mismo modo, para el levantamiento de información primaria se aplicaron entrevistas semi estructuradas a propietarios y administradores de los establecimientos, así como encuestas a clientes aplicando los cinco elementos para la valoración de la calidad del servicio de Servqual. 
Para la definición del objeto de estudio se consideraron factores de tipo geográfico y psicográfico para la definición del perfil del cliente, que permitieron identificar indicadores como; lugar de origen, nivel de instrucción, frecuencia de consumo, gasto promedio, entre otros, la valoración del nivel de satisfacción contemplo las cinco dimensiones de la calidad del servicio.

Una vez establecido el marco de estudio, se definió la población que en este caso corresponde a 30 establecimientos turísticos, de cinco sub sectores, siendo estos: alojamiento, alimentos y bebidas, intermediación, recreación y turismo comunitario, ubicados principalmente en la ciudad de Quito y provincias cercanas, estos establecimientos fueron seleccionados a partir del interés de los propietarios de participar en la investigación.

En la figura 1, se muestra la estructura de los establecimientos auditados, divididos en los cinco sub sectores turísticos.

Como se muestra en la figura 1 , el $44 \%$ de los establecimientos intervenidos corresponden al subsector de alojamiento, el $33 \%$ al de alimentos y bebidas (principalmente restaurantes), $13 \%$ a la intermediación (particularmente agencias de viajes y un empresa organizadora de eventos sociales), $7 \%$ al turismo comunitario (asociaciones comunitarias a nivel rural) y, 3\% a recreación, siendo en su totalidad Pymes.

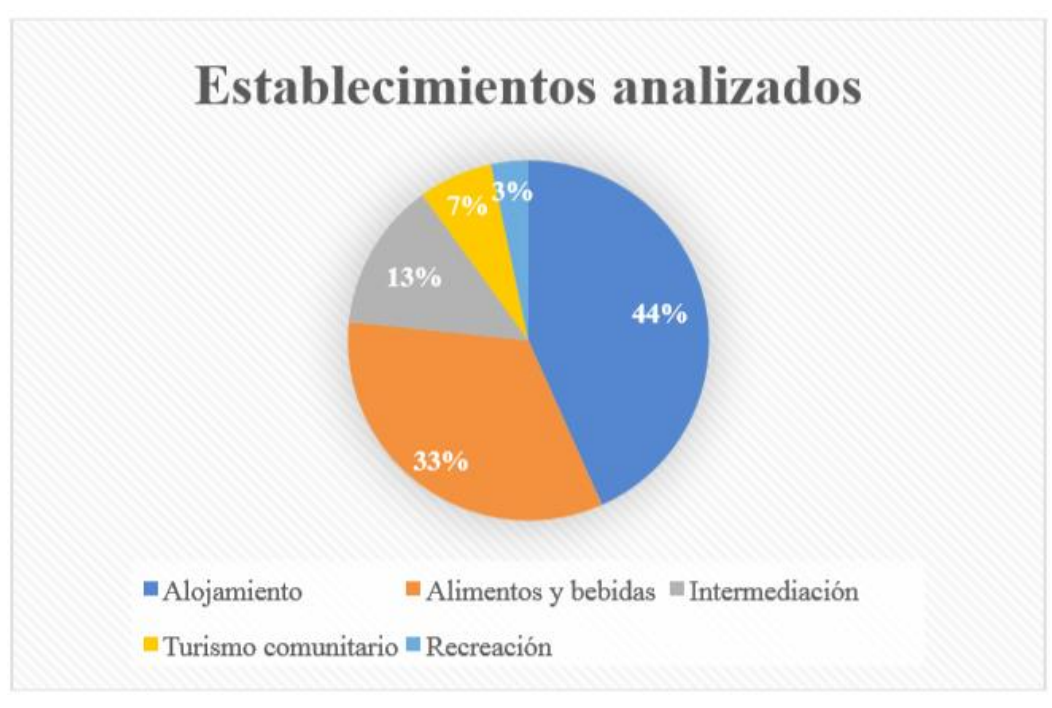

Figura 1. Estructura de los establecimientos analizados

Para la definición del tipo de muestreo este correspondió al tipo no probabilístico de forma aleatoria, el levantamiento de información se realizó de forma transversal por una sola vez, aplicando un cuestionario compuesto por 25 preguntas abiertas y cerradas de carácter cualitativo, en su mayoría usando escalas primarias nominales, así como con la aplicación de escalas métricas de intervalos (para la valoración del nivel de satisfacción se usó la escala de Likert), este cuestionario fue aplicado a los clientes de los establecimientos, (ver tabla 1). El procesamiento de la información fue realizado por medio del programa Excel, para medir el 
comportamiento de las variables de análisis, en este caso: planeación estratégica, identificación del perfil del cliente, aplicación de procesos de servicio y posibilidad de obtención de certificación.

Tabla 1. Ficha descripción metodológica

\begin{tabular}{ll}
\hline Población & 30 establecimientos \\
\hline Ámbito & $\begin{array}{l}\text { DM de Quito, Riobamba, Ibarra, Tulcán, Latacunga, } \\
\text { Santo Domingo de los Tsáchilas }\end{array}$ \\
Método para levantar datos & Encuesta personal \\
Respuesta a encuestas & Clientes de los establecimientos \\
Tamaño de la muestra & 25 encuestas por establecimiento \\
Tasa de respuesta & $100 \%$ \\
Margen de error & $8 \%$ \\
Nivel de confianza & $95 \%$ \\
Heterogeneidad & $50 \%$ \\
Trabajo de campo & Entre junio de 2014 y junio de 2017 \\
\hline & \multicolumn{1}{c}{ Elaboración propia }
\end{tabular}

\section{Resultados y Discusión}

Una vez aplicados los instrumentos de análisis se muestran los resultados iniciales obtenidos durante el periodo de análisis, como se mencionó se intervinieron 30 establecimientos correspondientes a; alojamiento (13), alimentos y bebidas (10), intermediación (4), turismo comunitario (2) y recreación (1). Los hallazgos principales muestran que; el 83,3\% de los establecimientos no poseen una adecuada planeación estratégica; el $90 \%$ no posee un adecuado organigrama y asignación de funciones; el 76.7\% desconoce el perfil de sus clientes.

Por otro lado, se valoró el grado de satisfacción del cliente, identificando los cinco criterios base identificados en el modelo Servqual, estos resultados fueron resumidos en una escala de valoración Likert, como se muestra en la siguiente figura.

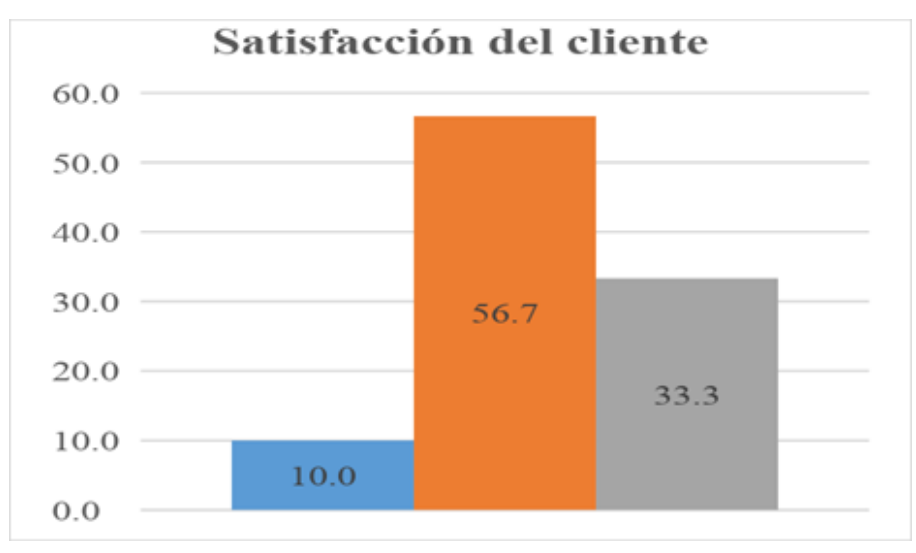


Figura 2. Valoración de la satisfacción del cliente.

Elaboración propia

Respecto al nivel de satisfacción del cliente en los establecimientos turísticos y de hospitalidad se identifica que, el 56.7\% se ubica en un rango medio (3), el 33,3\% medio alto (4), mientras que un $10 \%$ se ubica en un nivel bajo (2), cabe mencionar que del grupo objetivo de análisis, ninguno de los establecimientos obtuvo una valoración alta (5) correspondiente a "totalmente satisfecho", de igual manera, ningún establecimiento obtuvo una valoración baja (1) "totalmente insatisfecho", como se muestra en la figura 2.

Esto permite identificar a priori que no existiría una relación directa entre la existencia de una adecuada planeación estratégica por parte de los establecimientos y el grado de satisfacción de sus clientes, siendo necesario realizar un análisis estadístico comparativo que permita determinar dicha correspondencia entre ambas variables.

En un segundo momento, se valoró la existencia y aplicación de procesos de servicio en los establecimientos turísticos y de hospitalidad, arrojando los siguientes resultados:

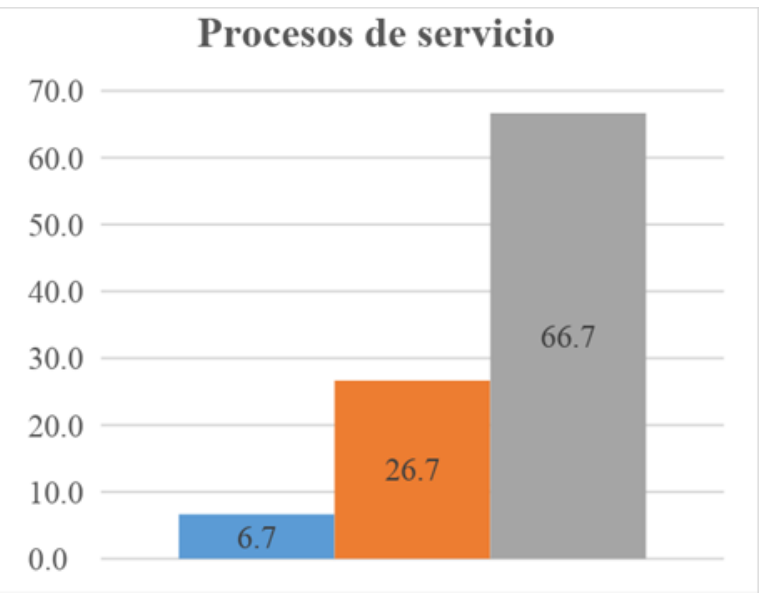

Figura 3. Existencia de procesos de servicio Elaboración propia

Como muestra la figura 3, el $66,7 \%$ de los establecimientos no posee o no aplica, procesos de servicio, mientras que un $26,7 \%$ posee algún tipo de protocolo o los aplican esporádicamente, frente al 6,67\% que posee y aplica adecuadamente sus protocolos de servicio.

Esta información da paso una nueva interrogante, ¿existe una relación directa entre el grado de satisfacción de los clientes y la aplicación de procesos de servicio?, como se mencionó esta investigación se encuentra en una fase inicial, en tal virtud, la comprobación de esta interrogante se realizará en una segunda fase de la investigación.

Finalmente, se valoró la posibilidad de obtener algún tipo de certificación de calidad o buenas prácticas, para ello se aplicaron listas de chequeo las cuales se adaptaron según el tipo de establecimiento y al sub sector que pertenecen, así por ejemplo, para el caso de turismo 
comunitario se aplicó un enfoque de buenas prácticas de sostenibilidad, mientras que para los establecimientos en zonas urbanas se aplicó la certificación SIGO o Distintivo Q, obteniendo los siguientes resultados.

La figura 3, muestra que del total de establecimientos valorados (30), el $80 \%$ no podría obtener una certificación o reconocimiento de calidad o sostenibilidad, al no alcanzar los mínimos requeridos por dichas certificaciones, por otro lado, un 6,7\% cumpliría con los mínimos requeridos para certificarse $y$, el restante $13,3 \%$ posee alguna certificación o reconocimiento nacional o internacional.

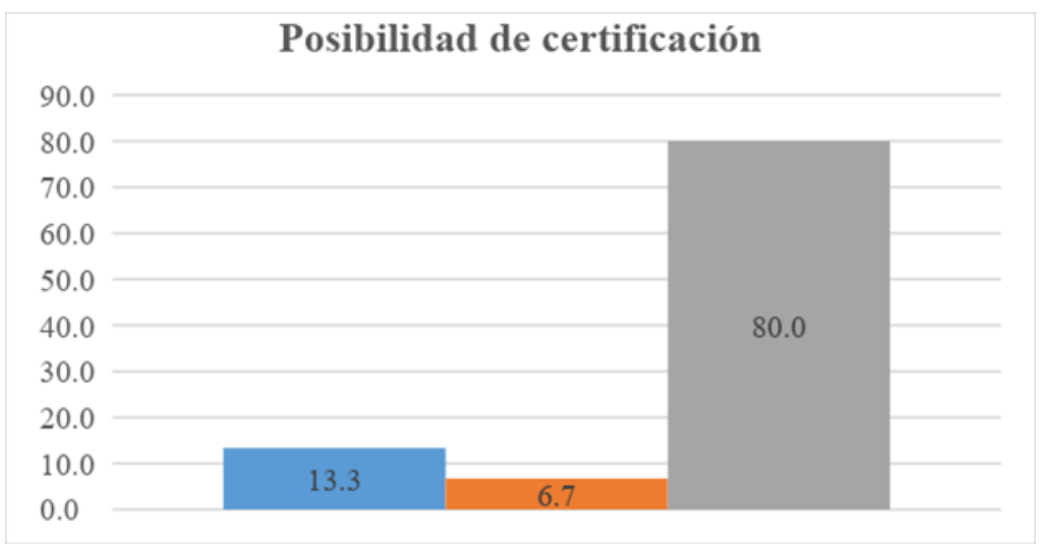

Figura 4. Posibilidad de obtener una certificación.

Elaboración propia

Si bien la certificación o reconocimiento de calidad, no es necesariamente un parámetro de medición de la calidad, o en su defecto la obtención de una certificación no representa la fase final de la misma, es un punto de partida para la consolidación de los procesos y el desarrollo de un valor diferenciador, eslabón final en el camino hacia la calidad. De la misma manera es necesario valorar si la inversión en la certificación genera algún tipo de beneficio directo a los establecimientos que opten por las mismas.

\section{Conclusión}

La calidad en el siglo veintiuno, se fundamenta principalmente en la satisfacción del cliente, el adecuado cumplimiento de los procesos, contar con una adecuada planeación y buscar permanentemente la innovación, es en este espacio en donde los servicios se convierten en el principal factor diferenciador, en un mercado cada vez más competitivo. Es por ello que las organizaciones deben prepararse para brindar una adecuada prestación del servicio. El modelo Servqual contempla una escala de valoración que permite tangibilizar elementos complejos como; la empatía, fiabilidad, capacidad de respuesta o la seguridad; en el sector turístico y de la hospitalidad la valoración de la calidad permite medir tanto los parámetros del servicio, como los de producto, pues resulta complejo identificar cuando inicia uno y termina otro.

Con los resultados obtenidos en este estudio, se identifica la debilidad que poseen los establecimientos turísticos y de la hospitalidad analizados, respecto a su planeación estratégica que, si bien no afectarían de forma directa a la prestación del servicio y al grado de satisfacción 
de los clientes, son cruciales para la consolidación de la organización. Otro elemento a considerar, es el desconocimiento del perfil del cliente y de su ventaja competitiva, ambas fundamentales respecto al posicionamiento de los establecimientos en el mercado turístico local e internacional.

A los elementos anteriores, se debe sumar la limitada implementación de adecuados procesos de operación, la inexistencia de estándares o el cumplimiento parámetros mínimos de servicio, esto por el contrario si son reflejados en el grado de satisfacción de los clientes que, si bien ubican su simpatía en un rango medio, no reflejan un posibilidad de fidelización o retorno, en tal virtud, los establecimientos analizados se encuentran lejos de cumplir o sobre pasar las expectativas del cliente.

Finalmente, se consideró la posibilidad de obtener una certificación de calidad o buenas prácticas, por parte de los establecimientos analizados, siendo esta bastante limitada, pues apenas 4 establecimientos cuentan con algún tipo de reconocimiento, los cuales coincidentemente poseen mejor manejo de procesos, una adecuada planeación estratégica, así como un mayor conocimiento de las necesidades de sus clientes, no es una casualidad entonces, que desde esta perspectiva la obtención de una certificación vaya de la mano con una mejora sustantiva de la organización, un mejor posicionamiento en el mercado y una mejora en su competitividad; como se mencionó, la obtención de una certificación no se convierte en la cúspide de la gestión de la calidad en el sector servicios, sin embargo, es un paso que debe ser considerado si lo que se busca es destacar en un mercado global creciente y altamente competitivo, en donde una adecuada prestación de los servicios, puede ser crucial a la hora de escoger un destino y la percepción posterior del cliente sobre el mismo.

\section{Bibliografía}

Albacete, C. A. (2004). Calidad de servicio en alojamientos rurales. Granada: Universidad de Granada.

Duque Oliva, E.J. Parra Díaz, M.C., (2015). Análisis de los modelos de medición de calidad percibida del servicio aplicados en la industria de hospitalidad. Perspectiva Empresarial. 2(2), 35-54. http://dx.doi.org/10.16967\%2Frpe.v2n2a4.

Escobar Valencia, M., \& Mosquera Guerrero, A. (2013). El marco conceptual relacionado con la calidad: una torre de Babel. Cuadernos de Administración, 29(50), 207-2016.

Garvin, D. A. (1988). Managing Quality: The Strategy and Competitive Edge. Nueva York: Free Press.

Grönroos, C. (1984). A service quality model and its marketing implications. European Journal of Marketing, 18(4), 36-44.

Grönroos, C. (1994). Marketing y gestión de servicios: la gestión de los momentos de la verdad y la competencia en los servicios. Madrid: Editorial Díaz de Santos. 
Kotler, P., Bowen, J., Makens, J., Rufín, R., \& Reina, M. D. (2004). Marketing para turismo (Pearson Educación). Madrid.

López, N. (2003). Marketing en restauración. Madrid: Editorial Síntesis. Miguel, J. A. (2002). Calidad de servicio en el sector turístico. Netbiblo.

Ministerio de Turismo. (2015, Mayo 26). MinTur entrega el distintivo "Q" de Calidad a establecimientos turísticos. Recuperado el 20 de noviembre de 2017, a partir de http://www.turismo.gob.ec/mintur-entrega-el-distintivo-q-de-calidad-a-establecimient osturisticos/

Ministerio de Turismo [Mintur]. (2015). Catastro Nacional de Establecimientos Turísticos 2015 (Sistema Integrado de Información Turística). Quito, Ecuador: Ministerio de Turismo.

Oliver, R. (1981). Measurement and evaluation of the satisfaction process in retail settings.

Journal of Retailing, 57, 25-48.

Parasuraman, A., Zeithaml, V., \& Berry, L. (1985). A conceptual model of service quality and its implications for future research. Journal of Marketing, 49(4), 41-45.

Salazar Duque, D. y Burbano Argoti, C. (2017). Análisis de la oferta gastronómica, una perspectiva comercial a través del marketing de servicios: Caso sector la Rumipamba, Pichincha, Ecuador. Revista Interamericana de Ambiente y Turismo. 13(1). 2-14. ISSN 0717-6651.

Santomá, R., \& Costa, G. (2007). Calidad de servicio en la industria hotelera: revisión de la Literatura. Revista de Análisis Turístico, (3), 27-44.

Secretaria Central de ISO. (2015). Norma Internacional ISO 9000: Sistemas de gestión de la calidad - Fundamentos y vocabulario. Secretaria Central de ISO. Recuperado a partir de

http://www.justicialarioja.gob.ar/planificacion/pagina/Norma\%20ISO\%209000_2015\%2

0Vocabulario\%20Fundamentos.pdf 